\title{
Analysis of EEG background activity in Alzheimer's disease patients with Lempel-Ziv complexity and Central Tendency
}

\section{Measure}

Daniel Abásolo, Roberto Hornero, Carlos Gómez, María García, Miguel López E.T.S. Ingenieros de Telecomunicación, University of Valladolid, Spain

AUTHOR'S ADDRESS: Daniel Abásolo

E.T.S. Ingenieros de Telecomunicación

University of Valladolid

Camino del Cementerio s/n

47011 - Valladolid (Spain)

Phone: +34983423981

Fax: +34983423667

E-mail: danaba@tel.uva.es 


\begin{abstract}
In this study we have investigated the electroencephalogram (EEG) background activity in patients with Alzheimer's disease (AD) using non-linear analysis methods. We calculated the Lempel-Ziv (LZ) complexity - applying two different sequence conversion methods - and the Central Tendency Measure $(C T M)$ of the EEG in 11 AD patients and 11 age-matched control subjects. CTM quantifies the degree of variability, while $L Z$ complexity reflects the arising rate of new patterns along with the EEG time series. We did not find significant differences between AD patients and control subjects' EEGs with CTM. On the other hand, AD patients had significantly lower $L Z$ complexity values $(p<0.01)$ at electrodes $\mathrm{P} 3$ and $\mathrm{O} 1$ with a two symbol sequence conversion, and P3, P4, O1 and T5 using three symbols. Our results show a decreased complexity of EEG patterns in AD patients. In addition, we obtained $90.9 \%$ sensitivity and $72.7 \%$ specificity at $\mathrm{O} 1$, and $72.7 \%$ sensitivity and $90.9 \%$ specificity at $\mathrm{P} 3$ and $\mathrm{P} 4$. These findings suggest that $L Z$ complexity may contribute to increase the insight into brain dysfunction in $\mathrm{AD}$ in ways which are not possible with more classical and conventional statistical methods.
\end{abstract}

Keywords: Alzheimer's disease; EEG; Non-linear dynamics; Lempel-Ziv Complexity; Central Tendency Measure. 


\section{Introduction}

Alzheimer's disease (AD) is the most common neurodegenerative disease and is considered to be the main cause of dementia in western countries [1]. AD is characterized by progressive impairments in cognition and memory whose course lasts several years prior to death. These clinical features are accompanied by characteristic histological changes in the brain. They include diffuse atrophy of the cortex and microscopical neuritic plaques (containing amyloid $A \beta$ ), neurofibrillary tangles and deposits of amyloid in the walls of the brain arteries. Although a definite diagnosis is only possible by necropsy, a differential diagnosis with other types of dementia and with major depression should be attempted. Magnetic resonance imaging and computerized tomography can be normal in the early stages of $\mathrm{AD}$ but a diffuse cortical atrophy is the main sign in brain scans. Mental status tests are also useful.

The electroencephalogram (EEG) has been used as a tool for diagnosing dementias for several decades. Typical EEG abnormalities in AD are characterized by a diffuse slowing of the background activity [2], namely through an increase in delta and theta activity along with a decrease in alpha activity, and a decreased coherence among cortical areas [3], although in the early stages of the disease the EEG may exhibit normal frequencies.

Recent progress in the theory of non-linear dynamics has provided new methods for the study of the EEG [4]. Non-linearity in the brain is introduced even at the cellular level, since the dynamical behaviour of individual neurons is governed by threshold and saturation phenomena. Moreover, the hypothesis of an entirely stochastic brain can be rejected due to its ability to perform sophisticated cognitive tasks. Considering this, non-linear dynamical analysis techniques may be a better approach than traditional linear methods to obtain a better understanding of abnormal dynamics in EEG signals [5,6].

Many studies are known in which non-linear time series analysis techniques were 
applied to EEGs. Correlation dimension $\left(D_{2}\right)$, a measure of system dimensional complexity [7], has been extensively investigated in EEG studies of different physiological and pathological states [8-13]. Particularly, several studies have examined the $D_{2}$ in AD. The idea behind them is that the loss of neurons and synapses results in less complex dynamics of neural networks and, consequently, reduced $D_{2}$ values [14]. It has been found that AD patients have lower $D_{2}$ values than controls [10,15-17]. These results show a decrease in the complexity of the electrical activity in brains injured by AD [4].

The Lyapunov exponents have also traditionally been used to characterize non-linear behaviour: if the first Lyapunov exponent $(L 1)$ is positive, the system is "chaotic" [18]. $L 1$ reflects the "unpredictability" of the underlying system and has been applied in EEG analysis to study the changes in normal brain development [19]. Moreover, it has been shown that AD patients have significantly lower $L 1$ values than controls in almost all EEG channels $[16,17]$. The decrease of $L 1$ in the EEG of AD patients reflects a drop in the flexibility of information processing in the injured brain [16].

Nevertheless, the amount of data required for meaningful results in the computation of $D_{2}$ and Lyapunov exponents is beyond the experimental possibilities for physiological data [20]. Moreover, the algorithms used to estimate the $D_{2}$ assume the time series to be stationary, something generally not true with biological data. Therefore, it becomes necessary to apply other non-linear methods to study the EEG background activity. For instance, non-linear forecasting and entropy maps have been used to characterize drug effects on brain dynamics in $\mathrm{AD}$ [21] and mutual information analysis to assess information transmission between different cortical areas in AD [22]. More recently, Pijnenburg et al. [23] have found a decrease of beta band synchronization likelihood both in a resting condition and during a working memory task in AD. 
In the present study, we have examined the EEG background activity in AD patients with two non-linear methods: Lempel-Ziv ( $L Z)$ complexity and computation of the Central Tendency Measure (CTM) from scatter plots of first differences of the data. We wanted to test the hypothesis that the AD patients' EEG might be characterized by an abnormal type of dynamics. The $L Z$ complexity [24] is a nonparametric measure of complexity in a onedimensional signal related to the number of distinct substrings and the rate of their recurrence. CTM is a non-linear approach using continuous chaotic modelling that summarizes the degree of variability in a signal [25].

The paper is organized as follows. In Section 2 we explain the selection of patients and controls, and the procedure for recording the EEG and selecting artefact-free epochs. $L Z$ complexity, CTM and the statistical tools used to evaluate the differences between AD patients and control subjects are also introduced in Section 2. Section 3 presents the results of our study. Finally, in Section 4 we discuss our results and compare them with other studies of the EEG background activity in AD patients with non-linear analysis methods, and we draw our conclusions.

\section{Methods}

\subsection{Selection of patients and controls}

We studied 11 patients $(5$ men and 6 women; age $=72.5 \pm 8.3$ years, mean \pm standard deviation SD) fulfilling the criteria of probable $\mathrm{AD}$. The patients were recruited from the Alzheimer's Patients' Relatives Association of Valladolid (AFAVA) and referred to the University Hospital of Valladolid (Spain), where the EEG was recorded. All of them had undergone a thorough clinical evaluation that included clinical history, physical and neurological examinations, brain scans and a Mini-Mental State Examination (MMSE), generally accepted as a quick and simple way to evaluate cognitive function [26]. Five 
patients had a MMSE score of less than 12 points, indicating a severe degree of dementia. The mean MMSE score for the patients was $13.1 \pm 5.9$ (Mean \pm SD). Two subjects were receiving lorapezam. With therapeutic doses, benzodiapzepines may enhance beta activity, although no prominent rapid rhythms were observed in the visual examination of these two subjects' EEGs. None of the other patients used medication that could be expected to influence the EEG.

The control group consisted of 11 age-matched, elderly control subjects without past or present neurological disorders ( 7 men and 4 women; age $=72.8 \pm 6.1$ years, mean $\pm \mathrm{SD}$ ). The MMSE score value for all control subjects was 30 .

The local ethics committee approved the study. All control subjects and all caregivers of the demented patients gave their informed consent for participation in the current study. An EEG was recorded from all patients and controls.

\subsection{EEG recording}

The EEGs were recorded from the 19 scalp loci of the international 10-20 system (channels Fp1, Fp2, F3, F4, C3, C4, P3, P4, O1, O2, F7, F8, T3, T4, T5, T6, Fz, Cz and Pz), with all electrodes referenced to the chin. Recordings were made with the subjects in a relaxed state and under the eyes-closed condition in order to obtain as many artefact-free EEG data as possible. More than five minutes of data were recorded from each subject using a Profile Study Room 2.3.411 EEG equipment (Oxford Instruments). Data were first processed with a low-pass hardware filter at $100 \mathrm{~Hz}$, then they were sampled at $256 \mathrm{~Hz}$ and digitised by a 12-bit analogue-digital converter.

The recordings were visually inspected by a specialist physician to reject artefacts. Thus, only EEG data free from electrooculographic and movement artefacts, and with minimal electromyographic (EMG) activity were selected. Afterwards, EEGs were organized 
in 5 second artefact-free epochs (1280 points) that were copied as ASCII files for off-line analysis on a personal computer. An average number of $30.0 \pm 12.5$ artefact-free epochs (Mean \pm SD) were selected from each electrode for each subject.

In order to remove the residual EMG activity and the noise owing to the electrical mains, all selected epochs were digitally filtered prior to the non-linear analysis. We used a Hamming window FIR band-pass filter with cut-off frequencies at $0.5 \mathrm{~Hz}$ and at $40 \mathrm{~Hz}$ designed with Matlab ${ }^{\circledR}$.

\subsection{Central Tendency Measure}

Chaotic equations are sometimes used to generate graphs. We can produce scatter plots of first differences of the data which graph $x(n+2)-x(n+1)$ versus $x(n+1)-x(n)$, where each $x(n)$ is the value of the EEG time series at time $n$. These plots, centred around the origin, give a graphical representation of the degree of variability in the time series and are useful in modelling biological systems such as hemodynamics and heart rate variability [25]. With this approach, rather than defining a time series as chaotic or not chaotic, the degree of variability or chaos is evaluated.

To quantify this level of variability, the central tendency measure (CTM) has been used [25]. CTM is computed from the scatter plots of first differences of the data selecting a circular region of radius $\rho$ around the origin, counting the number of points that fall within the radius, and dividing by the total number of points. A low CTM value indicates a large amount of dispersion and a high value concentration near the centre. Given $N$ data points from a time series, $N-2$ would be the total number of points in the scatter plot. Then, the CTM can be computed as [25], 


$$
C T M=\frac{\sum_{i=1}^{N-2} \delta\left(d_{i}\right)}{N-2}
$$

where

$$
\delta\left(d_{i}\right)=\left\{\begin{array}{l}
1 \text { if }\left[(x(i+2)-x(i+1))^{2}+(x(i+1)-x(i))^{2}\right]^{1 / 2}<\rho \\
0 \text { otherwise }
\end{array}\right.
$$

The application of this approach to a classification problem involving the separation of congestive heart failure patients from normal individuals analysing R-R intervals from Holter tapes shows promise [25]. Preliminary studies indicate that the method can be adapted to determine the clinical significance of the variability findings in more complex time series such as the EEG [27]. Moreover, the combination of CTM analysis of the EEG, clinical parameters and neuropsychological testing can be useful in the diagnosis of $\mathrm{AD}$ [28] and in the differentiation among types of dementia [29].

Although the radius is critical in determining the outcome of CTM, no guidelines exist for optimising its value. Hence, it is usually chosen depending upon the character of the data. We have developed a new method to select the radius $\rho$. First, we compute the CTM with several radii. Then, we apply a one-way ANOVA test to compare the CTM results of both groups and we estimate the $p$ value for each of the radii. The selected radius is that for which we obtain the lowest $p$ value in the comparison between both groups. In this study we have computed the $C T M$ with $\rho=2$.

\subsection{Lempel-Ziv Complexity}

The Lempel-Ziv (LZ) complexity for sequences of finite length was suggested by Lempel and Ziv [24]. It is a nonparametric, simple-to-calculate measure of complexity in a 
one-dimensional signal that does not require long data segments to compute [30]. $L Z$ complexity is related to the number of distinct substrings and the rate of their recurrence along the given sequence [31], with larger values corresponding to more complexity in the data. It has been applied to study the brain function [32], brain information transmission [33] and to detect ventricular tachychardia and fibrillation [30]. Preliminary evidence suggests that, applied to EEGs, $L Z$ complexity is predictive of epileptic seizures [31] and can be useful to quantify the depth of anaesthesia [6,34]. Moreover, it has been applied to extract complexity from mutual information time series of EEGs in order to predict response during isoflurane anaesthesia with artificial neural networks [35].

$L Z$ complexity analysis is based on a coarse-graining of the measurements, so before calculating the complexity measure $c(n)$, the signal must be transformed into a finite symbol sequence. In this study we have used two different sequence conversion methods:

a) 0-1 sequence conversion. The median value is estimated as a threshold $T_{d}$, as partitioning about the median is robust to outliers [36]. By comparison with the threshold, the signal data are converted into a $0-1$ sequence $P=s(1), s(2), \ldots, s(n)$, with $s(i)$ defined by [6]:

$$
s(i)= \begin{cases}0 & \text { if } x(i)<T_{d} \\ 1 & \text { if } x(i) \geq T_{d}\end{cases}
$$

b) 0-1-2 sequence conversion. For each of the EEG segments, the median $x_{m}$, maximum $x_{\max }$ and minimum $x_{\min }$ are calculated. Two thresholds are obtained: $T_{d l}=x_{m}-\left|x_{\min }\right| / 16$ and $T_{d 2}=x_{m}+\left|x_{\max }\right| / 16$ [6]. Then the EEG data are converted into a 0-1-2 sequence $P$ $=s(1), s(2), \ldots, s(n)$, with $s(i)$ defined by [6]:

$$
s(i)= \begin{cases}0 & \text { if } x(i) \leq T_{d 1} \\ 1 & \text { if } T_{d 1}<x(i)<T_{d 2} \\ 2 & \text { if } x(i) \geq T_{d 2}\end{cases}
$$


The sequence $P$ is scanned from left to right for both conversion methods and the complexity counter $c(n)$ is increased by one unit every time a new subsequence of consecutive characters is encountered. The complexity measure can be estimated using the following algorithm [6,30,34]:

1. Let $S$ and $Q$ denote two subsequences of $P$ and $S Q$ be the concatenation of $S$ and $Q$, while sequence $S Q \pi$ is derived from $S Q$ after its last character is deleted ( $\pi$ means the operation to delete the last character in the sequence). Let $v(S Q \pi)$ denote the vocabulary of all different subsequences of $S Q \pi$. At the beginning, $c(n)$ $=1, S=s(1), Q=s(2)$, therefore, $S Q \pi=s(1)$.

2. In general, $S=s(1), s(2), \ldots, s(r), Q=s(r+1)$, then $S Q \pi=s(1), s(2), \ldots, s(r)$; if $Q$ belongs to $v(S Q \pi)$, then $Q$ is a subsequence of $S Q \pi$, not a new sequence.

3. Renew $Q$ to be $s(r+1), s(r+2)$ and judge if $Q$ belongs to $v(S Q \pi)$ or not.

4. Repeat the previous steps until $Q$ does not belong to $v(S Q \pi)$. Now $Q=s(r+1)$, $s(r+2), \ldots, s(r+i)$ is not a subsequence of $S Q \pi=s(1), s(2), \ldots, s(r+i-1)$, so increase $c(n)$ by one.

5. Thereafter, $S$ is renewed to be $S=s(1), s(2), \ldots, s(r+i)$, and $Q=s(r+i+1)$.

These procedures have to be repeated until $Q$ is the last character. At this time the number of different subsequences in $P$ - the measure of complexity - is $c(n)$.

In order to obtain a complexity measure which is independent of the sequence length, $c(n)$ should be normalized. If the length of the sequence is $n$ and the number of different symbols in the symbol set is $\alpha$, it has been proved [24] that the upper bound of $c(n)$ is given by:

$$
c(n)<\frac{n}{\left(1-\varepsilon_{n}\right) \log _{\alpha}(n)}
$$


where $\varepsilon_{n}$ is a small quantity and $\varepsilon_{n} \rightarrow 0(n \rightarrow \infty)$. In general, $n / \log _{\alpha}(n)$ is the upper bound of $c(n)$, where the base of the logarithm is $\alpha$, i.e.,

$$
\lim _{n \rightarrow \infty} c(n)=b(n) \equiv \frac{n}{\log _{\alpha}(n)}
$$

and $c(n)$ can be normalized via $b(n)$ :

$$
C(n)=\frac{c(n)}{b(n)}
$$

$C(n)$, the normalized $L Z$ complexity, reflects the arising rate of new patterns along with the sequence. Thus, it captures the temporal structure of the sequence.

\subsection{Statistical analysis}

One-way ANOVA tests were used to evaluate the statistical differences between the estimated $L Z$ complexity and $C T M$ values for AD patients and control subjects. If significant differences between groups were found, the ability of the non-linear analysis method to discriminate $\mathrm{AD}$ patients from control subjects was evaluated using Receiver Operating Characteristic (ROC) plots [37].

ROC plots can be obtained by plotting the sensitivity values (the proportion of patients with a diagnosis of $\mathrm{AD}$ who test positive) on the $y$ axis against their equivalent (1-specificity) values (specificity represents the percentage of controls correctly recognized) for all the available cut-off points (in this case, the non-linear analysis method values) on the $x$ axis. Accuracy is a related parameter that quantifies the total number of subjects (AD 
patients and control subjects) precisely classified. The optimum threshold is the cut-off point in which the highest accuracy (minimal false negative and false positive results) is obtained. It can be determined from the ROC curve as the closest value to the left top point $(100 \%$ sensitivity, $100 \%$ specificity).

Figure 1 shows a block diagram with the different steps followed in this study.

Insert figure 1 around here

\section{Results}

$L Z$ complexity and $C T M$ were estimated for channels Fp1, Fp2, F3, F4, C3, C4, P3, P4, O1, O2, F7, F8, T3, T4, T5 and T6. The results have been averaged based on all the artefact-free 5 second epochs ( $N=1280$ points) within the five-minute period of EEG recordings.

The $C T M$ values (Mean $\pm \mathrm{SD}$ ) for the $\mathrm{AD}$ patients and control subjects and the corresponding $p$-values are summarized in Table 1 . No significant differences were found between both groups $(p>0.01)$.

Insert Table 1 around here

The average $L Z$ complexity values and standard deviations for the $\mathrm{AD}$ patients and normal control subjects for the 16 electrodes are summarized in Table 2 (0-1 sequence conversion) and Table 3 (0-1-2 sequence conversion). The AD patients have significantly lower $L Z$ complexity values $(p<0.01)$ at electrodes $\mathrm{P} 3$ and $\mathrm{O} 1$ for the two symbol sequence conversion, and electrodes $\mathrm{P} 3, \mathrm{P} 4, \mathrm{O} 1$ and $\mathrm{T} 5$ for the three symbol sequence conversion. 
These results suggest that EEG activity of $\mathrm{AD}$ patients is less complex in certain regions than in a normal brain.

Insert Tables 2 and 3 around here

Finally, we evaluated the ability of the $L Z$ complexity to discriminate AD patients from control subjects with both sequence conversions at the electrodes in which significant differences were found using ROC plots [37]. Table 4 summarizes the results.

Insert table 4 around here

The value for the area under the ROC curve can be interpreted as follows: an area of 0.893 (electrode P3 with 0-1-2 sequence conversion, for example) means that a randomly selected individual from the control subjects' group has a $L Z$ complexity value larger than that of a randomly chosen individual from the AD patients' group in $89.3 \%$ of the time [37]. A rough guide to classify the precision of a diagnostic test is related to the area under the ROC curve. With values between 0.90 and 1 the precision of the diagnostic test is considered to be excellent, good for values between 0.80 and 0.90 , fair if the results are in the range $0.70-0.79$, poor when the value of the area under the ROC curve is between 0.60 and 0.69 , and bad for values between 0.50 and 0.59 . Thus, the results obtained with $L Z$ complexity and both sequence conversion methods can be considered good.

With the $0-1$ sequence conversion the highest sensitivity was obtained at O1 (90.9\%) and the highest specificity at P3 (90.9\%). The accuracy of the diagnostic test was similar at both electrodes $(81.8 \%)$. Figure 2 shows the ROC curves for both electrodes. 
Insert figure 2 around here

Using a three symbol sequence conversion we obtained the highest specificity at P4 (90.9\%), while the highest sensitivity was obtained at O1 (90.9\%). The accuracy was $81.8 \%$ at P3, P4 and O1. Figure 3 shows the ROC curves for all the electrodes in which the differences between both groups were significant with the 0-1-2 sequence conversion.

Insert figure 3 around here

\section{Discussion}

In this pilot study we have analysed the EEG background activity of 11 control subjects and 11 patients with AD applying two non-linear methods: Lempel-Ziv (LZ) complexity and computation of the Central Tendency Measure (CTM) from scatter plots of first differences of the data.

The CTM method has proven not to be effective in discriminating AD patients from control subjects. We have not found relevant differences between the degree of variability from scatter plots of first differences of the EEG time series from both groups. However, our findings differ from other studies - with very small sample sizes - showing that the combination of CTM analysis of the EEG, clinical parameters and neuropsychological testing can be useful in the diagnosis of dementia [28,29]. Thus, the possible usefulness of CTM in the diagnosis of $\mathrm{AD}$ should be investigated with a larger number of patients and control subjects.

On the other hand, our results show that $\mathrm{AD}$ patients have significantly lower $L Z$ complexity values $(p<0.01)$ at electrodes P3 and O1 when we employed a two symbol 
sequence conversion. Moreover, using a three symbol sequence conversion we found a significantly reduced $L Z$ complexity $(p<0.01)$ at electrodes P3, P4, O1 and T5 in AD patients. Although previous studies claimed that a two symbol sequence conversion was enough to study the dynamic complexity of a system [31-33], our results suggest that a three symbol conversion might give more detailed insight of the differences between the AD patients and control subjects' EEGs. This may be due to the fact that a 0-1-2 conversion can keep more information of the EEG during the coarse-graining process. Furthermore, we compared our $L Z$ complexity results by means of sensitivity, specificity and accuracy with a selected threshold to improve the sensitivity/specificity pair according to ROC plots [37]. For both sequence conversion methods the highest sensitivity was obtained at electrode $\mathrm{O} 1$ (90.9\%), while the specificity improved in the parietal region $(90.9 \%$ at electrode P3 with a 01 sequence conversion and at electrode $\mathrm{P} 4$ with a 0-1-2 sequence conversion).

A larger value of $L Z$ complexity means that the chance of new pattern generation is greater, so the sequence is more complex, and vice versa. We infer that brains affected by AD show a less complex electrophysiological behaviour, specially in the left parietal and occipital regions. Our results agree with findings showing that the EEG of AD patients has lower $D_{2}$ values than that of control subjects $[14,16,17]$. In non-linear EEG analysis, $D_{2}$ is considered to be a reflection of the complexity of the cortical dynamics underlying EEG recordings. Thus, reduced $D_{2}$ values exhibit a decrease in the complexity of brain electrical activity. Furthermore, Besthorn et al. [38] found that a lower $D_{2}$ was correlated with increased severity of dementia and that this method correctly classified AD patients and controls with an accuracy of $70 \%$ [39]. Pritchard et al. [15] showed that the addition of non-linear measures $\left(D_{2}\right)$ and a neural net classification procedure to linear methods improves the classification accuracy of the AD/control status of subjects up to $92 \%$. The decreased complexity of brain activity in $\mathrm{AD}$ patients has also been shown using $L 1[16,17]$. The $L 1$ of the EEG can be 
interpreted as a measure of flexibility of information processing in the brain [40]. Thus, the decreased $L 1$ values in AD patients reflect a drop in the flexibility of information processing in the injured brain $[16,17]$.

However, the neurophysiological implications of the decreased EEG complexity in AD patients are not clear [4]. Among others, three mechanisms can be responsible for this decrease in dynamical complexity: neuronal death, a general effect of neurotransmitter deficiency and loss of connectivity of local neural networks as a result of nerve cell death [4,14]. The reduction of the $L Z$ complexity in the EEG of AD patients may be interpreted as "decomplexification" associated with the inactivation of previously active neural networks.

One important feature of $L Z$ complexity is model-independence. Only those differences between activity patterns that make a difference to the underlying system itself are considered, no matter whether the system is dominated by deterministic chaos or a stochastic process [6]. While applying this non-linear method to the EEG, we are not testing for a particular model form, but attempting to distinguish among the EEG data sets of AD patients and control subjects on the basis of complexity. Moreover, $L Z$ complexity is a more precise measure than $L 1$ for characterizing order or disorder [41] and is better suited for EEG analysis than $D_{2}$ [6]. The use of this quantitative complexity measure can help us gain a better insight into the system dynamics [6].

Some limitations of our study merit consideration. First of all, the sample size was small. As a result, our findings are preliminary and require replication in a larger patient population before any conclusion can be made of its clinical diagnostic value. Moreover, the detected decrease of EEG complexity is not specific to AD. It appears in several physiological and pathological states, including anaesthesia [6], schizophrenia [11], vascular dementia [17] and during seizure activity in epilepsy [31,42]. Ageing and age-related diseases often accompany a wide-ranging loss of physiological complexity [43]. Thus, although LZ 
complexity might be a helpful tool for recognition of $\mathrm{AD}$, further work must be carried out to examine non-linear EEG activity in other types of dementia.

In summary, although non-linear EEG analysis cannot yet be applied as a diagnostic tool, our findings show the possibility to analyse the dynamical behaviour of the brain in AD patients with $L Z$ complexity. Non-linear dynamics suggest that $\mathrm{AD}$ can be a dynamical disease which is characterized by changes in the qualitative dynamics of physiological processes [4]. We expect that non-linear analysis will give us a deeper understanding of the brain function in ways which are not possible with more classical and conventional statistical methods.

\section{Acknowledgements}

We would like to thank Pedro Espino (Hospital Universitario, Valladolid), who selected the patients and collected the EEG recordings. We are very grateful to María Luisa Baz, Ph.D., who kindly revised the English version of this paper. The authors are also thankful for the fruitful comments of the referees. This research has been supported by the grant project No.VA019/04 from the Junta de Castilla y León.

\section{References}

[1] Bird TD. Alzheimer's disease and other primary dementias. In: Braunwald E, Fauci AS, Kasper DL, Hauser SL, Longo DL, Jameson JL, editors. 'Harrison's Principles of Internal Medicine'. New York: The McGraw-Hill Companies Inc, 2001:2391-2399.

[2] Markand ON. Organic brain syndromes and dementias. In: Daly DD, Pedley TA, editors. 'Current Practice of Clinical Electroencephalography'. New York: Raven Press, 1990:401-423. 
[3] Jeong J. Nonlinear dynamics of EEG in Alzheimer's disease. Drug Dev Res 2002;56:5766.

[4] Jeong J. EEG dynamics in patients with Alzheimer's disease. Clin Neurophysiol 2004;115:1490-1505.

[5] Kantz H, Schreiber T. 'Nonlinear Time Series Analysis', Cambridge, England: Cambridge University Press, 1997.

[6] Zhang XS, Roy RJ, Jensen EW. EEG complexity as a measure of depth of anesthesia for patients. IEEE Trans Biomed Eng 2001;48:1424-1433.

[7] Grassberger P, Procaccia I. Measuring the strangeness of strange attractors. Physica D 1983;9:189-208.

[8] Babloyantz A, Destexhe A. Low-dimensional chaos in an instance of epilepsy. Proc Natl Acad Sci USA 1986;83:3513-3517.

[9] Babloyantz A, Destexhe A. The Creutzfeldt-Jakob disease in the hierarchy of chaotic attractors. In: Markus M, Müller S, Nicolis G, editors. 'From chemical to biological organization'. Berlin: Springer-Verlag, 1988: 307-316.

[10] Stam CJ, Jelles B, Achtereekte HAM, Rombouts SARB, Slaets JPJ, Keunen RWM. Investigation of EEG nonlinearity in dementia and Parkinson's disease. Electroenceph clin Neurophysiol 1995;95:309-317.

[11] Jeong J, Kim DJ, Chae J H, Kim SY, Ko HJ, Paik IH. Nonlinear analysis of the EEG of schizophrenics with optimal embedding dimension. Med Eng Phys 1998;20:669-676.

[12] Pijn JP, van Neerven J, Noest A, Lopes da Silva FH. Chaos or noise in EEG signals; dependence on state and brain site. Electroenceph clin Neurophysiol 1991;79:371381. 
[13] Ferri R, Alicata F, Del Gracco S, Elia M, Musumeci SA, Stefanini MC. Chaotic behavior of EEG slow-wave activity during sleep. Electroenceph clin Neurophysiol 1996;99:539-543.

[14] Jelles B, van Birgelen JH, Slaets JPJ, Hekster REM, Jonkman EJ, Stam CJ. Decrease of non-linear structure in the EEG of Alzheimer patients compared to healthy controls. Clin Neurophysiol 1999;110:1159-1167.

[15] Pritchard WS, Duke DW, Coburn KL, Moore NC, Tucker KA, Jann MW, Hostetler RM. EEG-based neural-net predictive classification of Alzheimer's disease versus control subjects is augmented by non-linear EEG measures. Electroenceph clin Neurophysiol 1994;91:118-130.

[16] Jeong J, Kim SJ, Han SH. Non-linear dynamical analysis of the EEG in Alzheimer's disease with optimal embedding dimension. Electroenceph clin Neurophysiol $1998 ; 106: 220-228$.

[17] Jeong J, Chae JH, Kim SY, Han SH. Nonlinear dynamic analysis of the EEG in patients with Alzheimer's disease and vascular dementia. J Clin Neurophysiol 2001;18:58-67.

[18] Wolf A, Swift JB, Swinney HL, Vastano JA. Determining Lyapunov exponents from a time-series. Physica D 1985;16:285-317.

[19] Meyer-Lindenberg A. The evolution of complexity in human brain development: an EEG study. Electroenceph clin Neurophysiol 1996;99:405-411.

[20] Eckmann JP, Ruelle D. Fundamental limitations for estimating dimensions and Lyapunov exponents in dynamical systems. Physica D 1992;56:185-187.

[21] Pezard L, Martinerie J, Varela FJ, Bouchet F, Guez D, Derousné C, Renault B. Entropy maps characterize drug effects on brain dynamics in Alzheimer's disease. Neurosci Lett $1998 ; 253: 5-8$. 
[22] Jeong J, Gore JC, Peterson BS. Mutual information analysis of the EEG in patients with Alzheimer's disease. Clin Neurophysiol 2001;112:827-835.

[23] Pijnenburg YAL, vd Made Y, van Cappellen van Walsum AM, Knol DL, Scheltens P, Stam CJ. EEG synchronization likelihood in mild cognitive impairment and Alzheimer's disease during a working memory task. Clin Neurophysiol 2004;115:1332-1339.

[24] Lempel A, Ziv J. On the complexity of finite sequences. IEEE Trans Inform Theory $1976 ; 22: 75-81$.

[25] Cohen ME, Hudson DL, Deedwania PC. Applying continuous chaotic modeling to cardiac signals. IEEE Eng Med Biol Mag 1996;15:97-102.

[26] Folstein MF, Folstein SE, McHugh PR. Mini-mental state. A practical method for grading the cognitive state of patients for the clinician. J Psychiatr Res 1975;12:189_ 198.

[27] Cohen ME, Hudson DL. New chaotic methods for biomedical signal analysis. Proceedings of the IEEE EMBS International Conference on Information Technology Applications in Biomedicine 2000;123-128.

[28] Cohen ME, Hudson DL. EEG analysis based on chaotic evaluation of variability. Proceedings of the $23^{\text {rd }}$ Annual EMBS International Conference 2001;3827-3830.

[29] Hudson DL, Cohen ME, Kramer M, Szeri A, Chang FL. Diagnostic implications of EEG analysis in patients with dementia. Proceedings of the $2^{\text {nd }}$ International IEEE EMBS Conference on Neural Engineering 2005;629-632.

[30] Zhang XS, Zhu YS, Thakor NV, Wang ZZ. Detecting ventricular tachycardia and fibrillation by complexity measure. IEEE Trans Biomed Eng 1999;46:548-555.

[31] Radhakrishnan N, Gangadhar BN. Estimating regularity in epileptic seizure time-series data. A complexity-measure approach. IEEE Eng Med Biol 1998;17:89-94. 
[32] Wu X, Xu J. Complexity and brain functions. Acta Biophysica Sinica 1991;7:103-106.

[33] Xu J, Liu ZR, Liu R, Yang QF. Information transformation in human cerebral cortex. Physica D 1997;106:363-374.

[34] Zhang XS, Roy RJ. Derived fuzzy knowledge model for estimating the depth of anesthesia. IEEE Trans Biomed Eng 2001;48:312-323.

[35] Huang L, Yu P, Ju F, Cheng J. Prediction of response to incision using the mutual information of electroencephalogram during anesthesia. Med Eng Phys 2003;25:321327.

[36] Nagarajan R. Quantifying physiological data with Lempel-Ziv complexity - Certain issues. IEEE Trans Biomed Eng 2002;49:1371-1373.

[37] Zweig MH, Campbell G. Receiver-Operating Characteristic (ROC) plots: a fundamental evaluation tool in clinical medicine. Clin Chem 1993;39:561-577.

[38] Besthorn C, Sattel H, Geiger-Kabisch C, Zerfass R, Förstl H. Parameters of EEG dimensional complexity in Alzheimer's disease. Electroenceph clin Neurophysiol 1995;95:84-89.

[39] Besthorn C, Zerfass R, Geiger-Kabisch C, Sattel H, Daniel S, Schreiter-Gasser U, Förstl H. Discrimination of Alzheimer's disease and normal aging by EEG data. Electroenceph clin Neurophysiol 1997;103:241-248.

[40] Röschke J, Fell J, Beckmann P. Non-linear analysis of sleep EEG data in schizophrenia: calculation of the principal Lyapunov exponent. Psychiatr Res 1995;56:257-269.

[41] Kaspar F, Schuster HG. Easily calculable measure for the complexity of spatiotemporal patterns. Phys Rev A 1987;36:842-848.

[42] Lehnertz K. Non-linear time series analysis of intracranial EEG recordings in patients with epilepsy - an overview. Int J Psychophysiol 1999;34:45-52. 
[43] Kyriazis M. Practical applications of chaos theory to the modulation of human ageing: nature prefers chaos to regularity. Biogerontology 2003;4:75-90. 


\section{Tables}

Table 1

The average $C T M$ values of the EEGs for the AD patients and control subjects for all channels.

\begin{tabular}{cccc}
\hline Electrode & $\begin{array}{c}\text { AD patients (Mean } \\
\pm \text { SD) }\end{array}$ & $\begin{array}{c}\text { Control subjects } \\
(\text { Mean } \pm \text { SD) }\end{array}$ & \begin{tabular}{c} 
Statistical analysis \\
\cline { 3 - 4 }
\end{tabular} \\
\hline F3 & $0.52 \pm 0.16$ & $0.63 \pm 0.14$ & 0.1050 \\
F4 & $0.54 \pm 0.15$ & $0.63 \pm 0.15$ & 0.1747 \\
F7 & $0.31 \pm 0.10$ & $0.44 \pm 0.14$ & 0.0205 \\
F8 & $0.33 \pm 0.14$ & $0.45 \pm 0.14$ & 0.0795 \\
Fp1 & $0.40 \pm 0.17$ & $0.50 \pm 0.14$ & 0.1623 \\
Fp2 & $0.41 \pm 0.15$ & $0.50 \pm 0.18$ & 0.1834 \\
T3 & $0.23 \pm 0.12$ & $0.31 \pm 0.17$ & 0.2036 \\
T4 & $0.21 \pm 0.11$ & $0.35 \pm 0.17$ & 0.0336 \\
T5 & $0.41 \pm 0.08$ & $0.38 \pm 0.19$ & 0.6844 \\
T6 & $0.40 \pm 0.13$ & $0.36 \pm 0.16$ & 0.5182 \\
C3 & $0.61 \pm 0.19$ & $0.69 \pm 0.17$ & 0.2898 \\
C4 & $0.58 \pm 0.19$ & $0.67 \pm 0.18$ & 0.2746 \\
P3 & $0.57 \pm 0.15$ & $0.55 \pm 0.21$ & 0.7932 \\
P4 & $0.57 \pm 0.14$ & $0.53 \pm 0.20$ & 0.6001 \\
O1 & $0.40 \pm 0.10$ & $0.33 \pm 0.18$ & 0.2097 \\
O2 & $0.41 \pm 0.10$ & $0.32 \pm 0.16$ & 0.1224 \\
\hline
\end{tabular}


Table 2

The average $L Z$ Complexity (0-1 sequence conversion) values of the EEGs for the AD patients and control subjects for all channels.

\begin{tabular}{cccc}
\hline Electrode & $\begin{array}{c}\text { AD patients (Mean } \\
\pm \mathrm{SD})\end{array}$ & $\begin{array}{c}\text { Control subjects } \\
(\text { Mean } \pm \text { SD) }\end{array}$ & \begin{tabular}{c} 
Statistical analysis \\
\cline { 4 - 4 }
\end{tabular} \\
\hline F3 & $0.35 \pm 0.06$ & $0.39 \pm 0.08$ & 0.2909 \\
F4 & $0.38 \pm 0.05$ & $0.38 \pm 0.08$ & 0.8025 \\
F7 & $0.39 \pm 0.06$ & $0.40 \pm 0.08$ & 0.7425 \\
F8 & $0.39 \pm 0.05$ & $0.41 \pm 0.07$ & 0.5213 \\
Fp1 & $0.33 \pm 0.09$ & $0.39 \pm 0.06$ & 0.0730 \\
Fp2 & $0.33 \pm 0.06$ & $0.37 \pm 0.09$ & 0.2264 \\
T3 & $0.47 \pm 0.09$ & $0.48 \pm 0.12$ & 0.8449 \\
T4 & $0.48 \pm 0.11$ & $0.47 \pm 0.10$ & 0.8539 \\
T5 & $0.39 \pm 0.08$ & $0.46 \pm 0.07$ & 0.0236 \\
T6 & $0.38 \pm 0.08$ & $0.46 \pm 0.08$ & 0.0521 \\
C3 & $0.39 \pm 0.08$ & $0.43 \pm 0.06$ & 0.2166 \\
C4 & $0.41 \pm 0.09$ & $0.43 \pm 0.05$ & 0.4098 \\
P3 & $0.35 \pm 0.07$ & $0.44 \pm 0.05$ & 0.0017 \\
P4 & $0.39 \pm 0.07$ & $0.44 \pm 0.05$ & 0.0124 \\
O1 & $0.39 \pm 0.07$ & $0.49 \pm 0.07$ & 0.0056 \\
O2 & $0.39 \pm 0.07$ & $0.47 \pm 0.08$ & 0.0214 \\
\hline
\end{tabular}

Significant group differences are marked with an asterisk. 
Table 3

The average $L Z$ Complexity (0-1-2 sequence conversion) values of the EEGs for the AD patients and control subjects for all channels.

\begin{tabular}{cccc}
\hline Electrode & $\begin{array}{c}\text { AD patients (Mean } \\
\pm \mathrm{SD})\end{array}$ & $\begin{array}{c}\text { Control subjects } \\
(\text { Mean } \pm \text { SD) }\end{array}$ & \begin{tabular}{c} 
Statistical analysis \\
\cline { 4 - 4 }
\end{tabular} \\
\hline F3 & $0.33 \pm 0.09$ & $0.37 \pm 0.08$ & 0.2319 \\
F4 & $0.37 \pm 0.06$ & $0.37 \pm 0.07$ & 0.8442 \\
F7 & $0.36 \pm 0.10$ & $0.39 \pm 0.07$ & 0.3801 \\
F8 & $0.36 \pm 0.09$ & $0.40 \pm 0.07$ & 0.2324 \\
Fp1 & $0.29 \pm 0.11$ & $0.37 \pm 0.08$ & 0.0710 \\
Fp2 & $0.31 \pm 0.07$ & $0.38 \pm 0.07$ & 0.0500 \\
T3 & $0.44 \pm 0.08$ & $0.47 \pm 0.10$ & 0.4397 \\
T4 & $0.45 \pm 0.09$ & $0.45 \pm 0.09$ & 0.9735 \\
T5* & $0.38 \pm 0.06$ & $0.45 \pm 0.06$ & 0.0072 \\
T6 & $0.35 \pm 0.10$ & $0.44 \pm 0.07$ & 0.0194 \\
C3 & $0.35 \pm 0.11$ & $0.42 \pm 0.05$ & 0.0810 \\
C4 & $0.33 \pm 0.13$ & $0.42 \pm 0.04$ & 0.0456 \\
P3* & $0.33 \pm 0.07$ & $0.43 \pm 0.04$ & 0.0005 \\
P4 & $0.34 \pm 0.06$ & $0.42 \pm 0.05$ & 0.0028 \\
O1 & $0.38 \pm 0.06$ & $0.47 \pm 0.06$ & 0.0029 \\
O2 & $0.37 \pm 0.06$ & $0.45 \pm 0.08$ & 0.0225 \\
\hline
\end{tabular}

Significant group differences are marked with an asterisk. 
Table 4

Test results for $L Z$ complexity with both sequence conversion methods on the channels in which the differences between both groups were significant. The optimum threshold to discriminate AD patients and control subjects is included.

\begin{tabular}{ccccccc}
\hline $\begin{array}{c}L Z \\
\begin{array}{c}\text { complexity } \\
\text { sequence } \\
\text { conversion }\end{array}\end{array}$ & Electrode & Threshold & Sensitivity & Specificity & Accuracy & $\begin{array}{c}\text { Area under } \\
\text { the ROC } \\
\text { curve }\end{array}$ \\
\hline \multirow{2}{*}{$0-1$} & P3 & 0.3995 & $72.7 \%$ & $90.9 \%$ & $81.8 \%$ & 0.876 \\
& O1 & 0.4538 & $90.9 \%$ & $72.7 \%$ & $81.8 \%$ & 0.851 \\
\hline \multirow{2}{*}{$0-1-2$} & P3 & 0.3962 & $81.8 \%$ & $81.8 \%$ & $81.8 \%$ & 0.893 \\
& P4 & 0.3485 & $72.7 \%$ & $90.9 \%$ & $81.8 \%$ & 0.843 \\
& O1 & 0.4411 & $90.9 \%$ & $72.7 \%$ & $81.8 \%$ & 0.851 \\
& T5 & 0.4160 & $72.7 \%$ & $72.7 \%$ & $72.7 \%$ & 0.802 \\
\hline
\end{tabular}




\section{Figure legends}

Figure 1. Block diagram summarizing the steps followed in this study, from signal recording to the statistical analysis of the $L Z$ complexity and $C T M$ results.

Figure 2. ROC curves for discriminating AD patients and control subjects with $L Z$ complexity (0-1 sequence conversion) at the electrodes in which $p<0.01$ ( $\mathrm{P} 3$ and $\mathrm{O} 1)$. The ROC curve is plotted using a dotted line, with its values marked with an asterisk. The interpolating parametric cubic spline curve is superimposed. (A) P3. (B) O1.

Figure 3. ROC curves for discriminating AD patients and control subjects with $L Z$ complexity (0-1-2 sequence conversion) at the electrodes in which $p<0.01(\mathrm{P} 3, \mathrm{P} 4, \mathrm{O} 1$ and $\mathrm{T} 5)$. The ROC curve is plotted using a dotted line, with its values marked with an asterisk. The interpolating parametric cubic spline curve is superimposed. (A) P3. (B) P4. (C) O1. (D) T5. 


\section{Conflict of interest statement}

There are no conflicts of interest that could inappropriately influence this research work. 
Figure 1

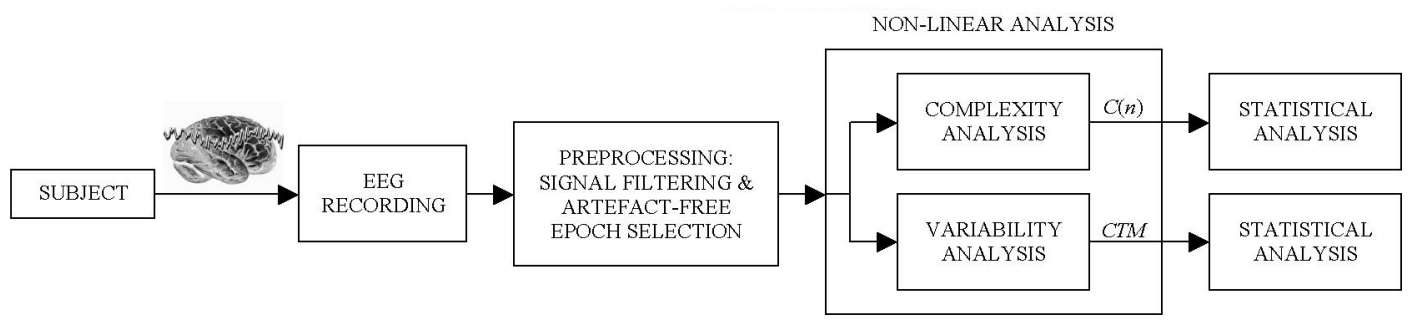


Figure 2

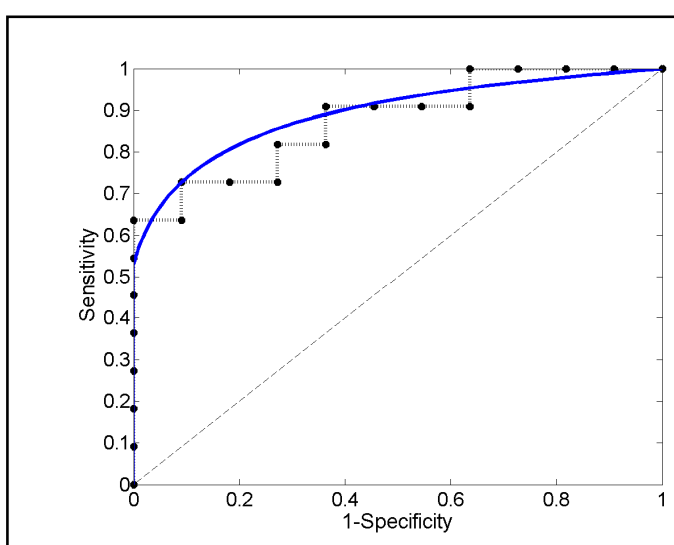

(a)

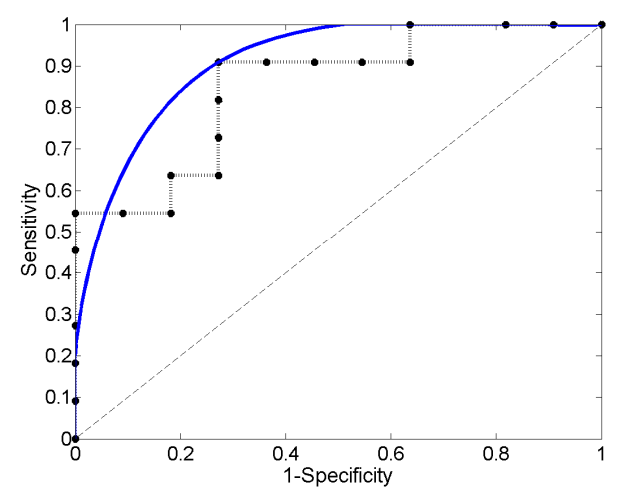

(b) 
Figure 3

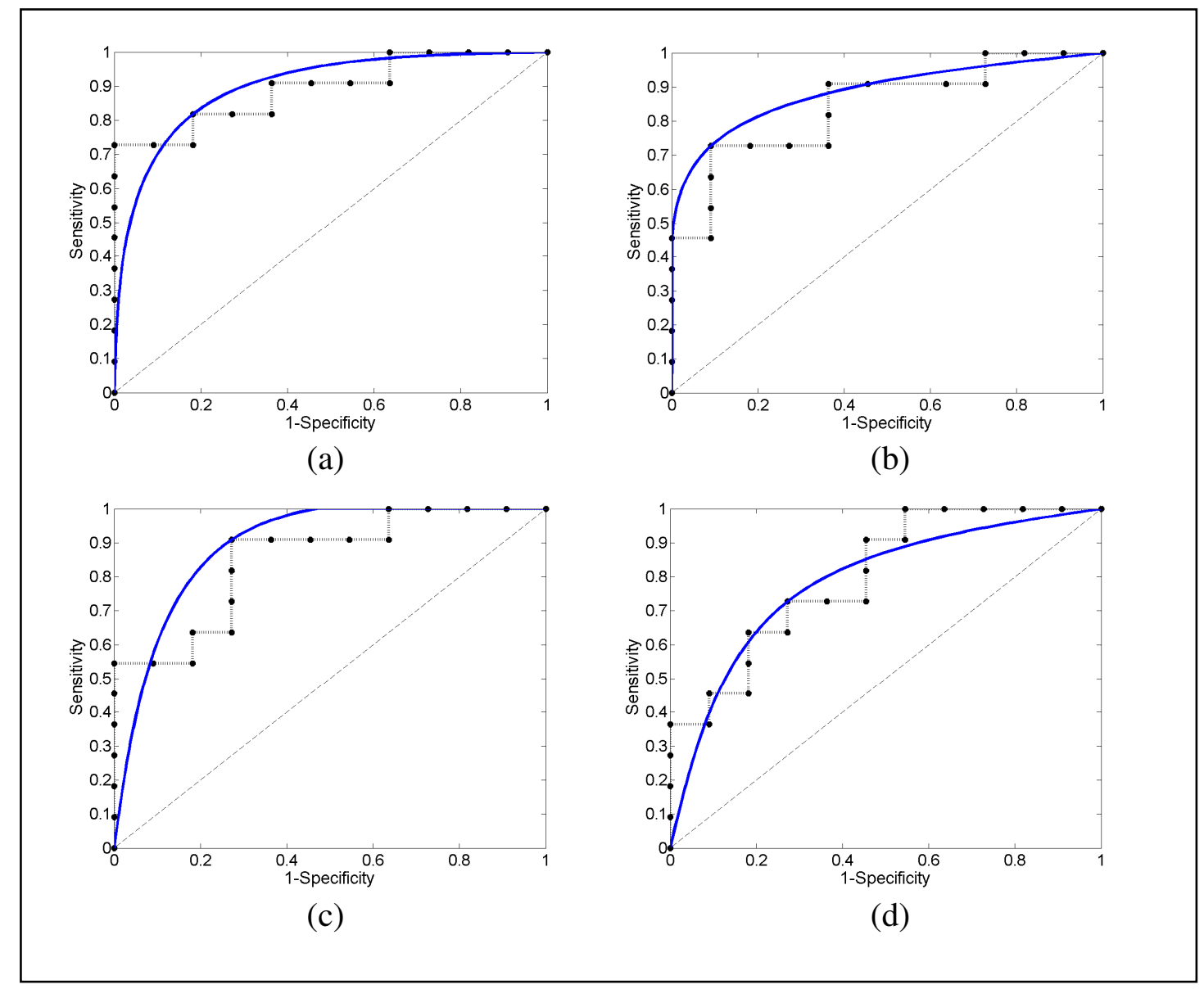

